\title{
A Novel Position and Velocity Observer for Robust Control of Switched Reluctance Motors
}

\author{
Y.J. Zhan, C.C. Chan and K.T. Chau \\ Department of Electrical \& Electronic Engineering, \\ The University of Hong Kong, Pokfulam Road, Hong Kong
}

\begin{abstract}
A new position and velocity sensorless control algorithm for the switched reluctance motor (SRM) drive is presented, which employs a sliding mode observer technique to estimate the motor speed and rotor position. This paper describes the construction of the observer, proposes the detailed method for designing the feed back gains of the observer and provides a quantitative robustness analysis against disturbances. The proposed sliding observer scheme is applied to a $4 \mathrm{~kW}$ SRM drive and the system performance is verified by computer simulation and experimental results.
\end{abstract}

\section{NOMENCLATURE}

$\mathbf{i}=\left[i_{1}, \ldots, i_{n}\right]^{\mathrm{T}} \quad$ Phase current vector

$\mathbf{U}_{s}=\left[\mathrm{U}_{1}, \ldots, U_{n}\right]^{\mathrm{T}}$ Phase voltage vector

$\Psi=\left[\psi_{1}, \ldots, \psi_{n}\right]^{\mathrm{T}}$ Flux linkage vector

$\mathbf{H}(\theta) \quad$ Reciprocal inductance

$r \quad$ Phase resistance

$\theta \quad$ Rotor position

$T_{e}$ and $T_{l} \quad$ Electromagnetic torque and load

torque

$\omega_{r} \quad$ Rotor speed

$B \quad$ Viscous friction coefficient

$J \quad$ Moment of inertia

\section{INTRODUCTION}

Generally switched reluctance motor (SRM) requires a rotor position sensor for commutation and current control. These position sensors, however, not only add complexity and cost to the system but also tend to reduce the reliability of the drive system. A considerable variety of methods have therefore been proposed to eliminate the position sensors/encodes in the literature[1]-[8].

One of principal methods in the subjects of indirect position sensing of SRM is wave form detection method. Since the unsaturated phase inductance of a SRM varies significantly between the aligned and unaligned positions of the stator and rotor poles, this method is based on the principle of inductance measurement in one of the un-energized phases as reported by [2],[6],[8]. Although this kind of sensorless opera- tion has eliminated the need for direct position sensing, it imposes difficulties on:

- the generation of negative torque by injecting a diagnostic pulse [6];

- limited resolution with speed increasing due to time delay by waiting for falling of previous diagnostic phase [2];

- added diagnostic electronic circuitry.

The other method employs the state observer theory which models the motor characteristics and calculates the position angle of rotor by using terminal measurements of phase voltages and currents [1],[7]. The pioneer research of Luenberger observer theory to SRM drive was made by Lumsdaine and Lang [7]. Although a linear observer theory to SRM drive had been successfully implemented, the non-linearity due to the speed perturbation was neglected by means of very large rotor inertia. As a result of this, the performance of the observer may not be fast enough for transient operation. On the other hand, an accurate knowledge of machine and load parameters is required by Lumsdaine and other type of Luenberger observers.

In this paper, a novel position and velocity sliding mode observer for SRM drive is proposed. Sliding mode controllers have been proposed for improving the robustness of the controllers [11]. The sliding observer potentially offers similar advantages, in particular inherent robustness of parameter uncertainty and easy implementation to nonlinear systems as SRM drive. Due to state reconstruction, this method avoids the need of added diagnostic circuitry without negative torque generation.

\section{DESCRIPTION OF SRM DRIVE}

In the state space, the dynamics of a SRM drive can be modeled with assumptions of omitting the mutual inductance and magnetic linearity as:

$$
\left\{\begin{array}{l}
\frac{d \Psi}{d t}=-\mathbf{r H}(\theta) \Psi+\mathbf{U}_{s}+\mathbf{w}_{\psi} \\
\frac{d \omega_{r}}{d t}=\frac{T_{e}-T_{l}}{J}-\frac{B}{J} \omega_{r}=-\frac{B}{J} \omega_{r}+\frac{T_{e}}{J}+w_{\omega} \\
\frac{d \theta}{d t}=\omega_{r}+w_{\theta} \\
\mathbf{i}=\mathbf{H}(\theta) \Psi
\end{array}\right.
$$


where $\mathbf{w}=\left(\mathbf{w}_{\psi}, w_{\omega}, w_{\ominus}\right)^{T}$ is disturbances due to parameters deviations and load, and $\mathbf{x}=\left(\begin{array}{lll}\Psi & \omega_{r} & \theta\end{array}\right)^{\mathrm{T}}$ as the state vector.

Sometimes $T_{l}$ is considered as known or zero as [7]. However, this assumption could lead to a totally invalid response since, in practice, the load torque does exist and is hardly known precisely even at steady state operation due to the speed perturbations. Therefore, the load torque $T_{l}$ is treated as external disturbance as in the paper. To implement the sliding observer theory to the nonlinear system (1), the output equation of (1) must be linearized. From all physical experiments, the $\boldsymbol{H}(\theta)$ can be well modeled by:

$H_{a}(\theta)=1437+1134 \cos \theta+412 \cos (2 \theta)$

Equation (2) may be linearized by expanding it into a Taylor series expansion about equilibrium point $\mathbf{x}_{0}$ :

$$
\begin{aligned}
i_{a}= & H_{a}(\theta) \psi_{a} \\
\cong & {\left[\frac{\partial H_{a}(\theta) \psi_{a}}{\partial \psi_{a}}, \frac{\partial H_{a}(\theta) \psi_{a}}{\partial \psi_{b}}, \frac{\partial H_{a}(\theta) \psi_{a}}{\partial \psi_{c}},\right.} \\
& \left.\frac{\partial H_{a}(\theta) \psi_{a}}{\partial \omega}, \frac{\partial H_{a}(\theta) \psi_{a}}{\partial \theta}\right]_{\mathbf{x}=\mathbf{x}_{a}} \cdot\left(\mathbf{x}-\mathbf{x}_{\circ}\right) \\
= & {\left[H_{a}\left(\theta_{0}\right), 0,0,0,-1134 \sin \left(\theta_{0}\right) \psi_{a 0}\right] \mathbf{x}+d_{a}=C_{a} \mathbf{x}+d_{a} }
\end{aligned}
$$

Similar to the other two phases, the output equation becomes:

$$
\begin{aligned}
& \mathbf{i}=\left(\begin{array}{l}
i_{a} \\
i_{b} \\
i_{c}
\end{array}\right)=
\end{aligned}
$$

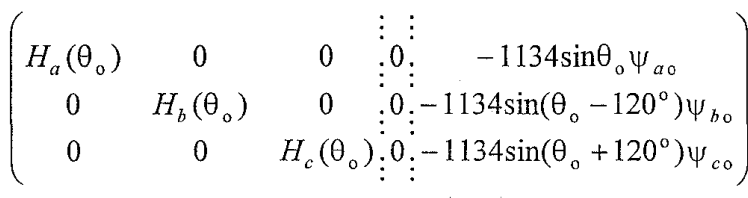

$$
\begin{aligned}
& \cdot \mathbf{x}+\left(\begin{array}{l}
d_{a} \\
d_{b} \\
d_{c}
\end{array}\right)=\left(\begin{array}{lll}
\mathbf{H}_{0} & 0 & \mathbf{C}_{0}
\end{array}\right) \mathbf{x}+\mathbf{D}=\mathbf{C} \mathbf{x}+\mathbf{D}
\end{aligned}
$$

where

$$
\begin{aligned}
& \mathbf{H}_{0}=\left(\begin{array}{ccc}
H_{a}\left(\theta_{0}\right) & 0 & 0 \\
0 & H_{b}\left(\theta_{0}\right) & 0 \\
0 & 0 & H_{c}\left(\theta_{o}\right)
\end{array}\right), \mathbf{D}=\left(d_{a}, d_{b}, d_{c}\right)^{\mathrm{T}}, \\
& \text { and } \mathbf{C}_{0}=\left(\begin{array}{c}
-1134 \sin \theta_{0} \psi_{a \circ} \\
-1134 \sin \left(\theta_{0}-120^{\circ}\right) \psi_{b o} \\
-1134 \sin \left(\theta_{0}+120^{\circ}\right) \psi_{c o}
\end{array}\right) \text { are constant }
\end{aligned}
$$

matrices.

\section{SLIDING MODE OBSERVER OF SRM DRIVE}

3.1 Sliding mode observer and system error dynamics

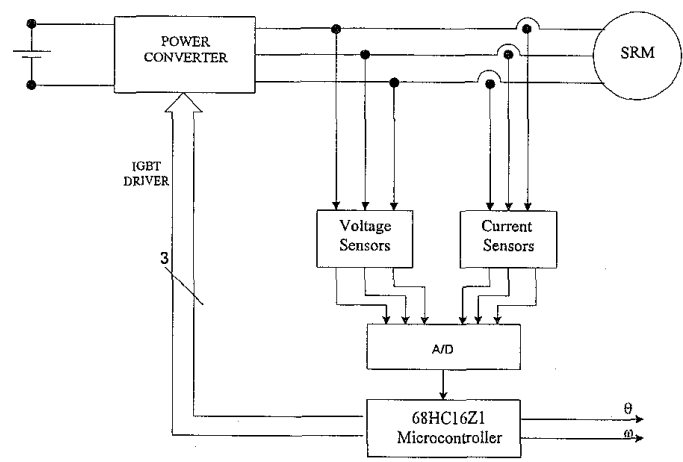

Fig. 1. System sensorless control configuration of SRM drive.

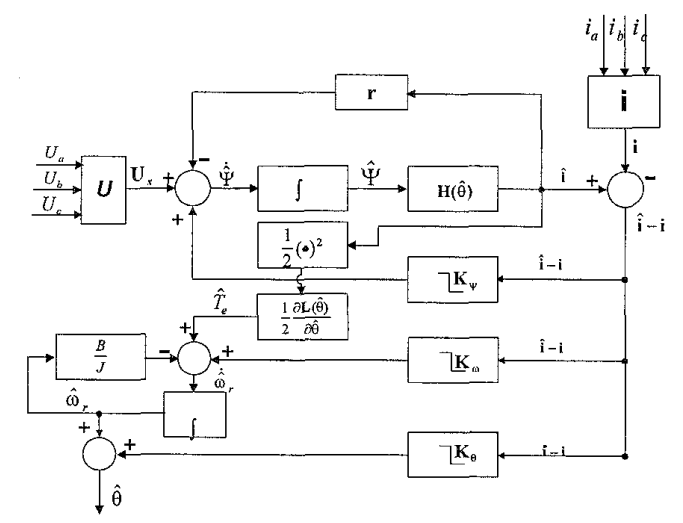

Fig. 2. The sliding observer configuration.

The sliding observer theory [9],[11] can directly be used to the dynamic model of SRM drive with the linearized output equation (4). The sliding observer for SRM drive can be constructed as:

$$
\left\{\begin{array}{l}
\frac{d \hat{\psi}}{d t}=-\mathbf{r H}(\hat{\theta}) \psi+\mathbf{U}_{s}+\mathbf{K}_{\psi} \operatorname{sgn}(\hat{\mathbf{i}}-\mathbf{i}) \\
\frac{d \hat{\omega}_{r}}{d t}=\frac{\hat{T}_{e}}{J}-\frac{B}{J} \hat{\omega}_{r}+\mathbf{K}_{\omega} \operatorname{sgn}(\hat{\mathbf{i}}-\mathbf{i}) \\
\frac{d \hat{\theta}}{d t}=\hat{\omega}_{r}+\mathbf{K}_{\theta} \operatorname{sgn}(\hat{\mathbf{i}}-\mathbf{i}) \\
\hat{\mathbf{i}}=\mathbf{H}(\hat{\theta}) \hat{\Psi}=\left(\begin{array}{lll}
\mathbf{H}_{0} & 0 & \mathbf{D}_{0}
\end{array}\right) \hat{\mathbf{x}}+\mathbf{C}
\end{array}\right.
$$

where ${ }^{\wedge}$ denotes estimated values. The present observer combines the SRM model (1) and with the additional innovation terms present in (5). The innovation terms are comparisons of the estimated and measured output currents multiplied by the switching gains $\mathbf{K}_{\psi}$, 
$\mathbf{K}_{\omega}$ and $\mathbf{K}_{\theta}$, which depend on $\hat{\Psi}, \hat{\omega}_{r}$ and $\hat{\theta}$ to reflect the principal nonlinearities of the SRM. Note that $\mathbf{K}_{\psi}=\operatorname{diag}\left(k_{\psi} \quad k_{\psi} \quad k_{\psi}\right) \quad$ is a matrix, whereas $\mathbf{K}_{\omega}=k_{\omega}\left(\begin{array}{lll}1 & 1 & 1\end{array}\right)$ and $\mathbf{K}_{\theta}=k_{\theta}\left(\begin{array}{lll}1 & 1 & 1\end{array}\right)$ are both row vector gains. Fig. 1 shows a block diagram of the SRM control system with a sliding observer. The sliding observer configuration is illustrated in Fig. 2. The inputs of the sliding mode observer are the source line-to-line voltages. The estimated three phase currents $\hat{\mathbf{i}}=\left(\begin{array}{lll}\hat{i}_{a} & \hat{i}_{b} & \hat{i}_{c}\end{array}\right)^{\mathrm{T}}$ are compared with the corresponding measured currents $\mathbf{i}=\left(\begin{array}{lll}i_{a} & i_{b} & i_{c}\end{array}\right)^{\mathrm{T}}$ and the differences of these currents are fed back through the sign functions. The errors of the system observer are defined as:

$\mathbf{e}_{\psi}=\hat{\Psi}-\Psi$

$e_{\omega}=\hat{\omega}_{r}-\omega_{r}$

$e_{\theta}=\hat{\theta}-\theta$

$\mathbf{e}_{\mathbf{i}}=\hat{\mathbf{i}}-\mathbf{i}=\left(\hat{i}_{a}-i_{a}, \hat{i}_{b}-i_{b}, \hat{i}_{\mathbf{c}}-i_{\mathbf{c}}\right)^{\mathrm{T}}$

Substitution of (1) and (5) into the derivatives of (6)(8) yields:

$$
\begin{aligned}
\frac{d \mathbf{e}_{\psi}}{d t} & =-\mathbf{r} \cdot \mathbf{e}_{i}+\mathbf{K}_{\psi} \operatorname{sgn} \mathbf{e}_{i}-\mathbf{w}_{\psi} \\
\frac{d e_{\omega}}{d t} & =-\frac{B}{J} e_{\omega}+\frac{1}{J}\left(\hat{T}_{e}-T_{e}\right)+\mathbf{K}_{\omega} \operatorname{sgn} \mathbf{e}_{i}-w_{\omega} \\
\frac{d e_{\theta}}{d t} & =e_{\theta}+\mathbf{K}_{\theta} \operatorname{sgn} \mathbf{e}_{i}+w_{\theta}
\end{aligned}
$$

as the observer error dynamics.

\subsection{Determination of switching gains $\mathbf{K}_{\psi}$ and $\mathbf{K}_{\theta}$}

To guarantee system sliding mode occur, the sliding hyperplane $\mathbf{s}$ on the state output $\mathbf{i}$ is defined as:

$$
\begin{aligned}
\mathbf{s} & =\mathbf{C}(\hat{\mathbf{x}}-\mathbf{x})=\left(\begin{array}{lll}
\mathbf{H}_{0} & \mathbf{0} & \mathbf{C}_{0}
\end{array}\right)\left(\begin{array}{lll}
\mathbf{e}_{\psi} & e_{\mathrm{\omega}} & e_{\theta}
\end{array}\right)^{\mathrm{T}} \\
& =\mathbf{H}_{0} \mathbf{e}_{\psi}+\mathbf{C}_{0} e_{\theta}
\end{aligned}
$$

and its derivative as:

$\dot{\mathbf{s}}=\mathbf{H}_{0} \dot{\mathbf{e}}_{\psi}+\mathbf{C}_{0} \dot{e}_{\theta}$

Sufficient condition of the sliding mode [9],[11] is expressed as:

$$
\begin{aligned}
& \mathbf{s}^{\mathrm{T}} \cdot \dot{\mathbf{s}}=\left(\mathbf{H}_{0} \mathbf{e}_{\psi}+\mathbf{C}_{0} e_{\theta}\right)^{\mathrm{T}}\left(\mathbf{H}_{0} \dot{\mathbf{e}}_{\psi}+\mathbf{C}_{0} \dot{e}_{\theta}\right) \\
& =\mathbf{e}_{\psi}^{\mathrm{T}} \mathbf{H}_{0}^{\mathrm{T}} \mathbf{H}_{0} \dot{e}_{\psi}+e_{\theta} \mathbf{C}_{0}^{\mathrm{T}} \mathbf{H}_{0} \dot{e}_{\psi}+\mathbf{e}_{\psi}^{\mathrm{T}} \mathbf{H}_{0}^{\mathrm{T}} \mathbf{C}_{0} \dot{e}_{\theta}+e_{\theta} \mathbf{C}_{0}^{\mathrm{T}} \mathbf{C}_{0} \dot{e}_{\theta}<0
\end{aligned}
$$

If the following inequalities:

$$
\begin{aligned}
& \mathbf{e}_{\psi}^{\mathrm{T}} \mathbf{H}_{0}^{\mathrm{T}} \mathbf{H}_{0} \dot{\mathbf{e}}_{\psi}<0 \\
& e_{\theta} \mathbf{C}_{0}^{\mathrm{T}} \mathbf{C}_{0} \dot{e}_{\theta}<0
\end{aligned}
$$

$e_{\theta} \mathbf{C}_{0}^{\mathrm{T}} \mathbf{H}_{0} \dot{\mathbf{e}}_{\psi}<0$

$\mathbf{e}_{\psi}^{\mathrm{T}} \mathbf{H}_{0}^{\mathrm{T}} \mathbf{C}_{0} \dot{e}_{\theta}<0$

is satisfied, then the sliding mode condition (15) can be fulfilled.

Since $\mathbf{H}_{0}>\mathbf{0}$ is valid and $\mathbf{D}$ is a varying value dependent upon the $\sin (\theta)$, the conditions of determining feedback gains can not be inferred by (18) and (19). Whereas (16) and (17) can be used for the deduction of the feedback gains. Hence substitution of (6) and (10) into the (16) yields:

$(\hat{\Psi}-\Psi)^{\mathrm{T}} \mathbf{H}_{0}^{\mathrm{T}} \mathbf{H}_{0}\left(-\mathbf{r e}_{i}+\mathbf{K}_{\psi} \operatorname{sgn}\left(\mathbf{e}_{i}\right)-\mathbf{w}_{\psi}\right)<0$

The switching gain $\mathbf{K}_{\psi}$ should be chosen so as to be large enough to satisfy (20) in order to generate the sliding mode. To the sliding observer, the sliding hyperplane is determined by the system itself whereas the switching gain $\mathbf{K}_{\psi}$ can be arbitrarily assigned to attain robustness against disturbances. However, a restriction on the assignment of the switching gain $\mathbf{K}_{\psi}$ comes from the requirement that the observer is stable, which can be expressed as that transmission zeros of the transfer function of observer must be on the open left halfplane. If the gain is too large a great amount of ripples must be contained, which may be a source of estimation errors. Therefore, a trade-off must be considered between the robustness and the stability. Since $\mathbf{i} \propto \Psi$ and the bound of disturbance $\mathbf{w}_{\psi}$ is known, $\mathbf{K}_{\psi}$ can be deduced from (20):

$\mathbf{K}_{\psi}<-\mathbf{r}\left\|\mathbf{e}_{\mathbf{i}}\right\|-\left\|\mathbf{w}_{\psi}\right\|$

where $\|$.$\| is quadratic norm of vector space. It is appar-$ ent that the gain is bounded by the current estimated error and parameters deviations. To an actual SRM system, these bounds are not difficult to be found.

To gain $\mathbf{K}_{\theta}=k_{\theta}\left(\begin{array}{lll}1 & 1 & 1\end{array}\right)$, similar substitution of $(8)$ and (12) into (17) yields:

$(\hat{\theta}-\theta) \mathbf{C}_{0}^{\mathrm{T}} \mathbf{C}_{0}\left(e_{\theta}+\mathbf{K}_{\theta} \operatorname{sgn}\left(\mathbf{e}_{i}\right)-w_{\theta}\right)<0$

From (22), if $\hat{\theta}-\theta>0$, then $e_{\theta}+\mathbf{K}_{\theta} \operatorname{sgn}\left(\mathbf{e}_{i}\right)-w_{\theta}<0$ must be valid. Since $\mathbf{i} \propto \theta^{-1}$ is tenable on a vicinity of the sliding plane, $\operatorname{sgn}\left(\mathbf{e}_{i}\right)<0$ is valid. Therefore $k_{\theta}$ must satisfy:

$k_{\theta}>e_{\mathrm{\omega}}-w_{\theta}$

Contrarily if $\hat{\theta}-\theta<0$, therefore:

$k_{\theta}>-e_{\omega}+w_{\theta}$

To satisfy both of (23) and (24), a uniform ultimate of gain $k_{\theta}$ can be obtained as:

$k_{\theta}>\left|e_{\omega}\right|+\left|w_{\theta}\right|$

It is apparent that the obtained $k_{\theta}$ satisfies inequality (22). 
3.3 The $H_{\infty}$ optimization of switching gain $\mathbf{K}_{\omega}$

Since $\boldsymbol{K}_{\omega}$ does not appear in the inequality of (15), the switching gain $\mathbf{K}_{0}$ can not be determined with above method. However on a vicinity of the sliding surface and during sliding mode, the switching term $\mathbf{K}_{\mathbf{0}}$ in (5) is acting to $\operatorname{keep} \mathbf{s}=(\hat{\mathbf{i}}-\mathbf{i})=\mathbf{0}$ and $\dot{\mathbf{s}}=(\dot{\hat{\mathbf{i}}}-\dot{\mathbf{i}})=\mathrm{C}(\dot{\hat{\mathbf{x}}}-\dot{\mathbf{x}})=\mathrm{C} \dot{\hat{\mathbf{e}}}=\mathbf{0}$, then:

$\dot{\mathbf{e}}_{\psi}=\mathbf{0}$

By substituting (26) into (10) and omitting the small voltage drop term, it can result:

$\dot{\mathbf{e}}_{\psi}=\mathbf{K}_{\psi} \operatorname{sgn}(\hat{\mathbf{i}}-\mathbf{i})-\mathbf{w}_{\psi}=\mathbf{0}$

and rearrangement of (27) results in:

$\mathbf{K}_{\psi} \operatorname{sgn}(\hat{\mathbf{i}}-\mathbf{i})=\mathbf{w}_{\psi}$

Defining $\mathbf{K}_{\omega}=\mathbf{L}_{\omega} \mathbf{K}_{\psi}$ and substituting it into (11), then (11) can be expressed as:

$\dot{\mathbf{e}}_{\omega}=-\frac{B}{J} \mathbf{e}_{\omega}+\mathbf{L}_{\omega} \mathbf{K}_{\psi} \operatorname{sgn}(\hat{\mathbf{i}}-\mathbf{i})+\frac{1}{J}\left(\hat{T}_{e}-T_{e}\right)-w_{\omega}$

Substitution of (28) into (29) results in:

$\dot{\mathbf{e}}_{\omega}=-\frac{B}{J} \mathbf{e}_{\omega}+\mathbf{L}_{\omega} \mathbf{w}_{\Psi}+\frac{1}{J}\left(\hat{T}_{e}-T_{e}\right)-w_{\omega}$

By defining:

$w_{\omega}^{\prime}=-\frac{1}{J}\left(\hat{T}_{e}-T_{e}\right)+w_{\omega}$

then error equation (30) becomes:

$\dot{\mathbf{e}}_{\omega}=-\frac{B}{J} \mathbf{e}_{\omega}+\mathbf{L}_{\omega} \mathbf{w}_{\Psi}+w_{\omega}^{\prime}=-\frac{B}{J} \mathbf{e}_{\omega}+\mathbf{F w}$

where $\mathbf{F}=\left(\begin{array}{ll}\mathbf{L}_{\omega} & 1\end{array}\right)$ and $\mathbf{w}=\left(\begin{array}{ll}\mathbf{w}_{\psi} & w_{\omega}^{\prime}\end{array}\right)^{\mathrm{T}}$.

For a realizable and stable system, the effects of all kind of disturbances can be analyzed by introducing $H_{\infty}$-norm of its transfer matrix. Through optimization of the norm, an optimum switching gain under different running conditions can be obtained. In the Hardy spaces $H_{\infty}$, the $\|T(s)\|_{\infty}$-norm is defined by:

$\|T(s)\|_{\infty}=\sup _{\omega} \sigma_{\max }(T(j \omega))$

where $\sigma_{\max }[T(j \omega)]$ is the maximum singular number of the matrix $T(j \omega)$, which can be obtained by:

$\sigma_{\max }[T(j \omega)]=\left[\lambda_{\max }\left(T^{*}(j \omega) T(j \omega)\right)\right]^{1 / 2}$

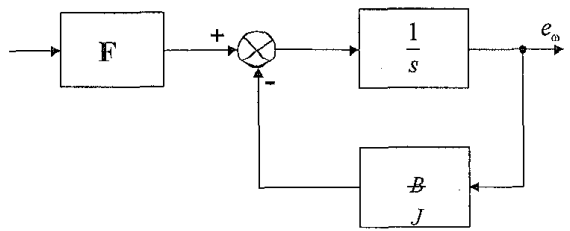

Fig. 3. Transfer function of the error system $e_{\omega}$. where $j^{2}=-1, T^{*}(j \omega)$ is a transpose and an adjoint matrix of $T(j \omega)$ and $\lambda_{\text {max }}$ is the maximum eigenvalue of the matrix $T^{*}(j \omega) T(j \omega)$. It can be seen from (33) that the $H_{\infty}$-norm represents the gain of $T(\mathrm{~s})$ corresponding to the worst disturbance. Therefore the $H_{\infty}$ norm in practice is criteria of error system performance in a sense of robustness of disturbance. From Fig. 3, the transfer matrix from disturbance $\mathbf{w}$ to error output $e_{\omega}$ is expressed as:

$\mathbf{T}_{w e}(s)=\left(s+\frac{B}{J}\right)^{-1} \cdot \mathbf{F}$

where $\mathrm{F}$ is disturbance input matrix defined by (32). From (33) and (34), the $\|T(s)\|_{\infty}$ is obtained as:

$\left\|\mathbf{T}_{w e}\right\|_{\infty}=\sup _{\omega} \sigma_{\max }\left[\mathbf{T}_{\mathbf{w}}(j \omega)\right]=\|\mathbf{F}\|_{2} \cdot J / B$

where $\|\mathbf{F}\|_{2}=\left(\mathbf{L}_{\omega}^{2}+1\right)^{1 / 2}$ is the induced quadratic norm of $\mathbf{F}$, and $\left\|T_{w e}\right\|_{\infty}$ can be seen as the maximum gain of the error system. For the worst disturbances $\mathbf{W}_{\text {max }}(s)$, the maximum error response $E_{\omega \max }(s)$ is obtained as:

$E_{\mathrm{a} \max }(s)=\left\|\mathbf{T}_{w e}\right\|_{\infty} \cdot \mathbf{W}_{\max }(s)$

To any given constant $\alpha>0$, the maximum error response $E_{\omega \max }(s)$ can be kept within bound of $\alpha$, or:

$E_{\text {o } \max }=\frac{J}{B}\|\mathbf{F}\|_{2} \cdot\left\|\mathbf{W}_{\max }\right\|_{2} \leq \alpha$

According to the Cauchy-Schwarz inequality [12]:

$|\langle\mathbf{F}, \mathbf{W}\rangle| \leq\|\mathbf{F}\|_{2} \cdot\|\mathbf{W}\|_{2}$

here $|\langle\mathbf{F}, \mathbf{W}\rangle|$ is inner product, then (38) is written as:

$\left[\left(\mathbf{L}_{\omega} \mathbf{w}_{\psi}\right)^{2}+\left(w_{\omega}^{\prime}\right)^{2}\right]^{1 / 2} \leq\left(\frac{\alpha B}{J}\right)$

From (40), an $H_{\infty}$-optimal observer can be obtained under existence of various system disturbances caused by parameter deviations and load. The situations are discussed as follows:

1. $\mathbf{r} \rightarrow(\mathbf{r}+\Delta \mathbf{r})$

$\mathbf{w}_{\psi}=-\Delta \mathbf{r} \mathbf{H}(\theta) \Psi$ and $\boldsymbol{w}_{\omega}^{\prime}=-\frac{\left(\hat{T}_{e}-T_{e}\right)+T_{l}}{J}$.

With 1., an $H_{\infty}$-optimal $\mathbf{L}_{\omega}$ can be obtained as:

$$
\begin{aligned}
& \left(\begin{array}{lll}
L_{\omega a} & L_{\omega b} \quad L_{\omega c}
\end{array}\right) \leq \frac{\left[(\alpha B)^{2}+\left(\hat{T}_{e}-T_{e}+T_{i}\right)^{2}\right]^{1 / 2}}{J} \\
& \left(\frac{1}{\left|\Delta r_{a}\right| i_{a}} \frac{1}{\left|\Delta r_{b}\right| i_{b}} \frac{1}{\left|\Delta r_{c}\right| i_{c}}\right)
\end{aligned}
$$


2. $\mathbf{H} \rightarrow(\mathbf{H}+\Delta \mathbf{H})$ :

$\mathbf{w}_{\psi}=-\mathbf{r} \Delta \mathbf{H}(\theta) \Psi$ and $\boldsymbol{w}_{\omega}^{\prime}=-\frac{\left(\hat{T}_{e}-T_{e}\right)+T_{l}}{J}$.

Substitution of 2. into (40) yields:

$\left(L_{\omega a} \quad L_{\omega b} \quad L_{\omega c}\right) \leq \frac{\left[(\alpha B)^{2}-\left(\hat{T}_{e}-T_{e}+T\right)^{2}\right]^{1 / 2}}{J}$

$\left(\frac{1}{r_{a}\left|\Delta H_{a}\right| \psi_{a}} \frac{1}{r_{b}\left|\Delta H_{b}\right| \psi_{b}} \frac{1}{r_{c}\left|\Delta H_{c}\right| \psi_{c}}\right)$

3. $B \rightarrow(B+\Delta B)$ :

$w_{\omega}^{\prime}=-\frac{\left(\hat{T}_{e}-T_{e}\right)+T_{l}}{J}-\frac{\Delta B}{J} \omega$ and $w_{\psi}=0$.

Using a similar method to the above, an $H_{\infty}$-optimal $\mathbf{L}_{\omega}$ under 3. can be obtained as:

$\left(-w_{\omega}^{\prime}\right)^{2} \leq\left(\frac{\alpha B}{J}\right)^{2}$

It is noted that (43) is independent on $\mathbf{L}_{\omega}$, hence it can be chosen arbitrarily large without violating the constrain of $H_{\infty}$, however large $\mathbf{L}_{0}$ trends to give dynamically an undesirable error. The $\mathrm{L}_{\omega}$ will be finally determined to have the uniform ultimate of gain under the most severe situation among conditions of $1 ., 2$. and 3 . without violating the sliding condition.

\section{SIMULATION AND EXPERIMENTAL RESULTS}

Table 1. System Parameters of SRM drive.

\begin{tabular}{ll}
\hline Power & $4 \mathrm{~kW}$ \\
Phase & 3 \\
Stator poles & 12 \\
Rotor poles & 8 \\
DC voltage & $240 \mathrm{~V}$ \\
Rated current & $40 \mathrm{~A}$ \\
Rated torque & $12.8 \mathrm{Nm}$ \\
Stator resistance & $0.3 \Omega$ \\
Reciprocal inductance & $1437+1134 \cos (\theta)(1 / \mathrm{H})$ \\
Viscous friction coefficient & $0.0012 \mathrm{Nms}^{2}$ \\
\hline
\end{tabular}

From above analyses, the optimal feedback gains $\mathbf{K}_{\psi}, \mathbf{K}_{\omega}$ and $\mathbf{K}_{\theta}$ can be expressed by (21), (25), (41) and (42) for the sliding observer (5). The adequacy of the proposed control algorithm was verified by simulations tests with a tested machine of a 3 phase $4-\mathrm{kW}$ SRM drive. The parameters of the SRM drive system are listed as Table 1 . In the simulations, some assumptions were made as follows:

- the initial conditions are $\hat{\Psi}(0)=\mathbf{0} ; \hat{\theta}(0)=0 ; \hat{\mathbf{i}}(0)=\mathbf{0}$ and $\hat{\omega}(0)=3 \mathrm{rad} / \mathrm{s}$ for overcoming the initial transient;
- the applied voltage and line current are detected exactly without time delay;

- in the simulation of the converter, the dead time and turn on/off time of IGBTs are taken into account.
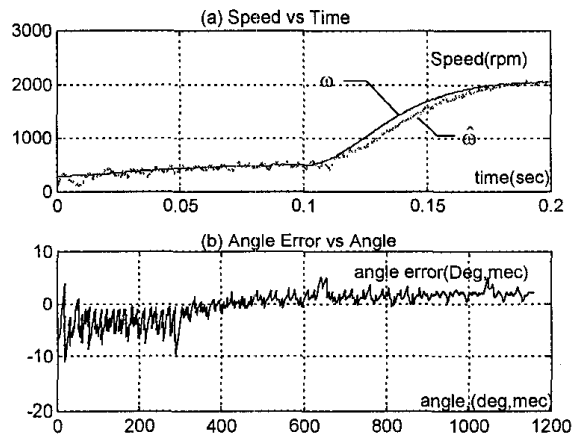

Fig. 5. Observer adjusted speed dynamic errors: (a) speed. (b) position. $\Delta \theta=10.5^{\circ}, \Delta n=300 \mathrm{rpm}, n^{*}=500 \mathrm{rpm}$ at $t=0 \mathrm{~s}$, then $2000 \mathrm{rpm}$ at $0.1 \mathrm{~s}$.
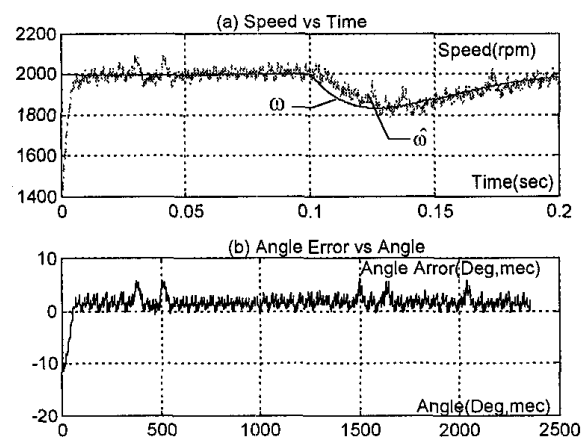

Fig. 6. Observer errors due to load disturbance: (a) speed. (b) position. $\Delta \theta=11.5^{\circ}, \Delta n=300 \mathrm{rpm}$ and $T_{l}=5 \mathrm{Nm}$ at $t=0 \mathrm{~s}$, then 7.5 $\mathrm{Nm}$ at $0.1 \mathrm{~s}$.
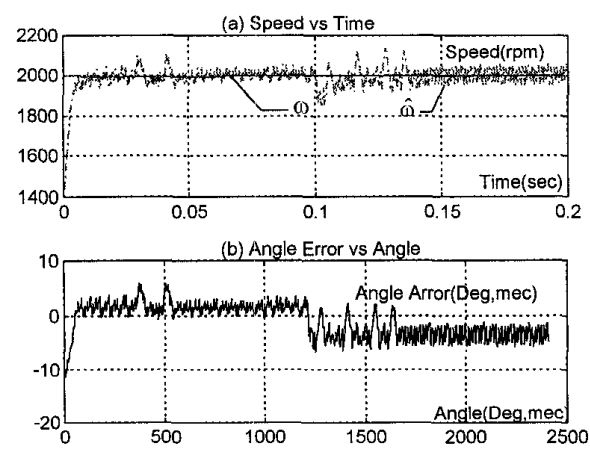

Fig. 7. Observer errors due to voltage deviation: (a) speed. (b) position. $\Delta \theta=11.5^{\circ}, \Delta n=500 \mathrm{rpm}$ and $V=240 \mathrm{~V}$ at $t=0 \mathrm{~s}$, then $200 \mathrm{~V}$ at $0.1 \mathrm{~s}$. 
The system was tested with the observer running from an arbitrary initial rotor position $\theta_{0}$ at standstill similar to the case when an opto-sensor is used. The simulation test results are shown in Fig. 5 through Fig. 7.

Fig. 5 displays the observer dynamic response errors of estimated rotor position and motor speed during acceleration. The results shows that the estimated positions are able to track the actual values and the sliding observer does not suffer from the nonlinearity of the torque characteristics. The system dynamic response error to an abrupt step-load change is shown in Fig. 6. Although additional errors in both of speed and position were generated, system could fast recover the track of actual values. Fig. 7 shows the insensitivity of the observer to supplied voltage variation. Although the abrupt variation of system voltage causes peak error, this effect did not spoil the overall drive performance and the errors was able to be kept at reasonable values.

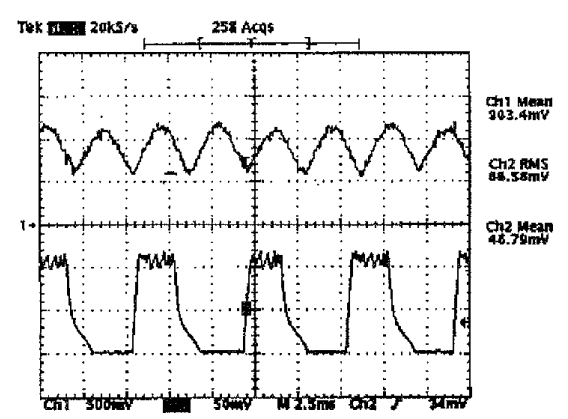

Fig. 8. Voltage (top) and phase current (bottom) waveforms at $1200 \mathrm{rpm}$. Voltage scale: $125 \mathrm{v} / \mathrm{div}$, current scale: $10 \mathrm{~A} / \mathrm{div}$.

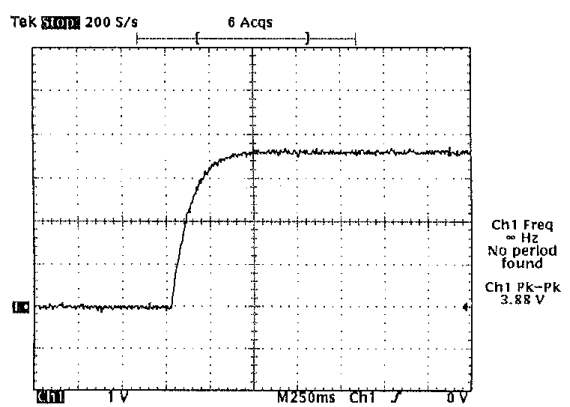

Fig. 9. Starting dynamic response with the sliding observer.

The practical realization of the observer algorithm requires a great amount of computation. Fig. 1 shows whole configuration implementation of the speed sensorless controller based on the Motorola MC68HC16Z1 microcontroller. The microcontroller consists of a digital signal processor (DSP) unit which can perform 32-bit floating-point multiplication with main frequency $16.78 \mathrm{MHz}$ and has the machine cycle for every calculation is $150 \mathrm{~ns}$. The sliding observer was run on-line at a sampling rate $50 \mu$ s. The controller also generates suitable current PWM and angles control signals with a $5 \mathrm{~ms}$ sampling time.

The Fig. 8 shows the oscillograph of the measured system bus voltage and chopped current flowing phase A with the integrated observer scheme, which was taken on a light load and at $1200 \mathrm{rpm}$ of the motor speed. The trace illustrates that the proposed observer performed a satisfied position estimation and angle signal firing. Fig. 9 verifies speed track trajectories of SRM drive system from $0-1500 \mathrm{rpm}$ with the position observer scheme.

\section{CONCLUSION}

The development of a sliding mode observer for SRM sensorless control is proposed and tested. The construction of the sliding observer is simple to implement by properly choosing switching gains. Stability of the scheme is assured and quantitative analysis for choosing switching gains against the system disturbances and parameter deviations are presented. Staring the motor from an arbitrary rotor position apart from its actual position, the observer is able to hunt the transient responses with a reasonable estimated errors. The feasibility and robustness of the proposed observer are supported by the simulation and experiment results. Further research will be done to implement the sliding observer to show its efficacy under various severe load conditions.

\section{ACKNOWLEDGMENT}

This work was supported and funded in part by the Committee on Research and Conference Grants, the University of Hong Kong.

\section{REFERENCES}

[1] C. Elmas and H.Z. Parra, "Position sensorless operation of a switched reluctance drive based on observer," EPE Conference Proceedings, pp. 8287, 1993.

[2] M. Ehsani, S. Mahajan, K.R. Ramani and I. Husain, "New modulation encoding techniques for indirect rotor position sensing in switched reluctance motors," IEEE-IAS Conference Record, pp. 430-438, 1992.

[3] Y. Liao and C. Sun, "A novel position sensorless control scheme for doubly fed reluctance motor drives," IEEE Transactions on Industry Applications, Vol. 30, No. 5, pp. 1210-1218, 1994. 
[4] R. Lagerquist, I. Boldea and T.J.E. Miller, "Sensorless control of the synchronous reluctance motor," Proceedings of IEEE-IAS Annual Meeting, pp. 427-436, 1993.

[5] F. Nozari, P. A. Mezs, A. L. Julian, C. Sun and T. A. Lipo, "Sensorless synchronous motor drive for use on commercial transport airplanes," IEEE Transactions on Industry Applications, Vol. 31, No. 4, pp. 850-859, 1995.

[6] S.R. MacMinn, W.J. Rzesos, P.M. Szczesny, and T.M. Jahns, "Application of sensor integration techniques to switched reluctance motor drives," Proceedings of IEEE-IAS Annual Meeting, pp. 584-588, 1988.

[7] A. Lumsdaine and J.H. Lang, "State observers for variable reluctance motors", IEEE Transactions on Industry Electronics, Vol. 37, No. 22, pp. 133$142,1990$.

[8] M. Ehsani, and I. Husain, "Rotor position sensing in switched reluctance motor drives by measuring mutually induced voltages," Proceedings of IEEE-IAS Annual Meeting, pp. 422-429. 1992.

[9] J.J. Slotine, J.K. Hedrick and E.A. Misawa, "On sliding observer for nonlinear systems," Journal of Dynamic Systems, Measurement, and Control, Vol. 109, pp. 245-252, 1987.

[10] K.D. Young, "Controller design for a manipulator using theory of variable structure systems," IEEE Transactions Systems, Man, Cybernetics, Vol. 8, No. 2, pp. 101-109, 1978.

[11] V.I. Utkin, "Principle identification with sliding observer," Dokl. Nauk SSSR, Vol. 257, No.3, pp. 558-561, 1981.

[12] W. L. Brogan, "Modern Control Theory," Prentice Hall, NJ, 1991. 